Review

\title{
Understanding laser beam brightness: A review and new prospective in material processing
}

\author{
Pratik Shukla ${ }^{\mathrm{a}, *}$, Jonathan Lawrence ${ }^{\mathrm{a}}$, Yu Zhang ${ }^{\mathrm{b}}$ \\ ${ }^{a}$ Laser Engineering and Manufacturing Research Group, Faculty of Science and Engineering, Thornton Science Park, University of Chester, Chester CH2 4NU, \\ United Kingdom \\ ${ }^{\mathrm{b}}$ University of Lincoln, Brayford Pool, Lincoln School of Engineering, Lincoln LN6 7TS, United Kingdom
}

\section{A R T I C L E I N F O}

Article history:

Received 22 January 2015

Received in revised form 23 May 2015

Accepted 3 June 2015

Keywords:

Brightness

Radiance

Luminance

Lasers

Materials processing

\begin{abstract}
A B S T R A C T
This paper details the importance of brightness in relation to laser beams. The 'brightness' of lasers is a term that is generally not given much attention in laser applications or in published literature. With this said, it is theoretically and practically an important parameter in laser-material processing. This study is first of a kind which emphasizes in-depth, the concept of brightness of lasers by firstly reviewing the existing literature and the progress with high brightness laser-material processes. Secondly, the techniques used to enhance the laser beam brightness are also reviewed. In addition, we review the brightness fundamentals and rationalize why brightness of lasers is an important concept. Moreover, an update on the analytical technique to determine brightness using the current empirical equations is also provided. A modified equation to determine the laser beam brightness is introduced thereafter. Furthermore, research studies previously conducted to modify laser design to affect laser beam brightness are also discussed. The paper not only involves a review of the techniques used to improve laser beam brightness but also reviews how bright lasers can be employed to enhance/improve laser process capabilities and cost reduction of the laser assisted processes in manufacturing.
\end{abstract}

(c) 2015 Elsevier Ltd. All rights reserved.

\section{Contents}

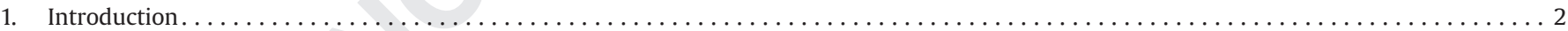

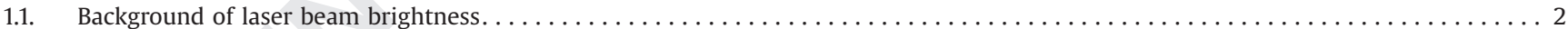

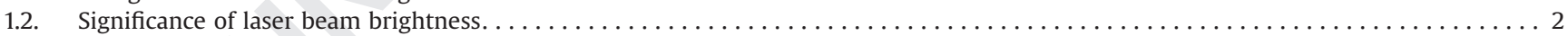

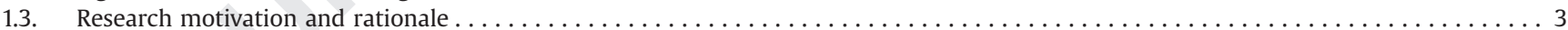

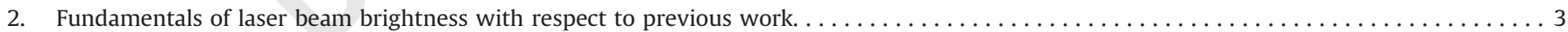

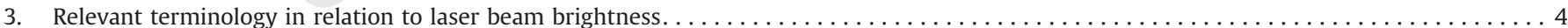

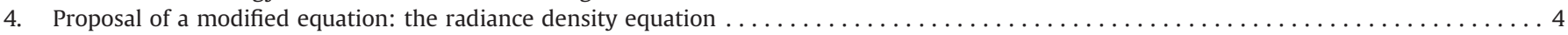

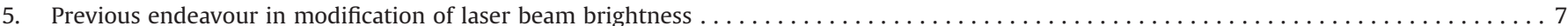

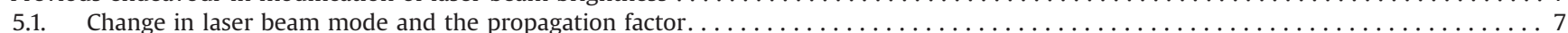

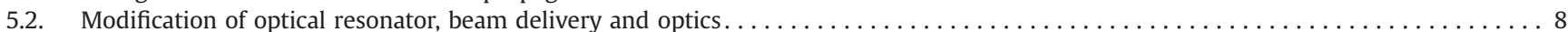

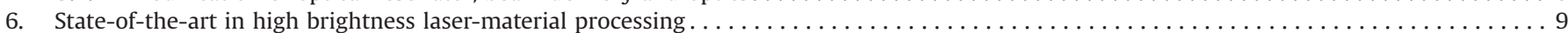

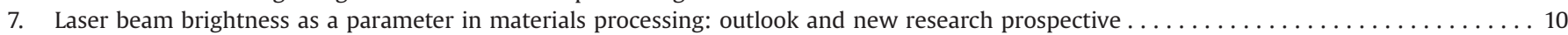

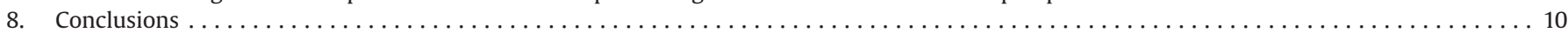

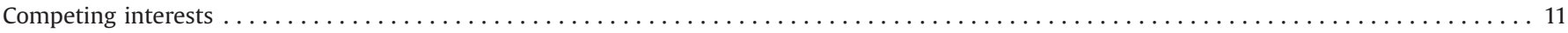

\footnotetext{
* Corresponding author.

E-mail address: pratik.shukla@talk21.com (P. Shukla).
}

http://dx.doi.org/10.1016/j.optlastec.2015.06.003

0030-3992/৫ 2015 Elsevier Ltd. All rights reserved. 


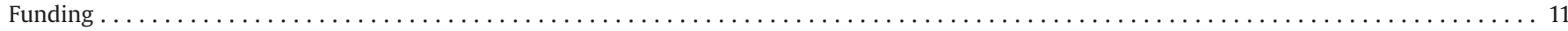

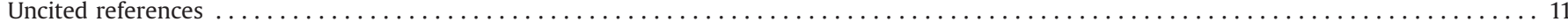

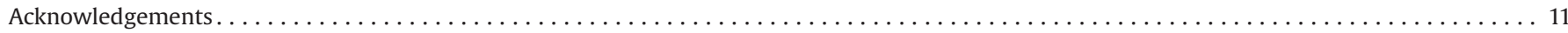

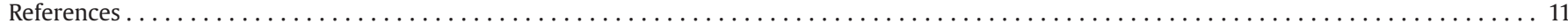

\section{Introduction}

\subsection{Background of laser beam brightness}

Brightness is a visual perception of light. The brightness of objects or a light source varies with change in the distance and the angle at which the object is visualized. Brightness also varies depending on the reflection, absorption and transmission of a light beam in relation to the surface in contact [1]. Thus, it could be defined as the amount of light in form of 'luminosity' observed on an object per unit of area [2,3]. Luminosity defines the measure of brightness, though such a term is rather suitable for only selected areas in science. As such, each discipline defines luminosity with its own term. Brightness is classified in two ways: one is photometric and the other is radiometric. The former quantifies light in terms of the judgement made by the human eye in assessing the brightness of an object, whereas, the latter quantifies brightness in terms of absolute power [1,4]. The photometric light refers to luminance and its luminosity. On the other hand, the radiometric light refers to 'radiance'. Both luminance and radiance, however, are so-called, cousins and have similarity in the sense that they ultimately relate to the brightness of a light source. However, luminance refers to contrast, glair and eye sensitivity to a particular wavelength. Luminance adheres to the principle that brighter the object - the more power it emits, and the higher the temperature of light (in relation to a particular distance). Comparably, radiance refers to a specific measure irrespective of eye sensitivity and wavelength of the light source and is dependent on power emitted per unit area in the solid angle of light divergence.

One of the first reported literature in relation to brightness was on the measurement of brightness and its colour over the sky by Reesincks in 1946 [5]. Reesincks developed a technique to obtain measurements of brightness during the summer period of 1946, in the Netherlands. Later, Zhang et. al. [6] reported on the feasibility of cloud remote sensing by ground based sky thermal infra-red brightness temperature measurement [6]. However, brightness in relation to lasers was a concept developed in the early 1960s [7]. Earlier work referring to a specified light source such as a laser was much focused on enhancing and improving the capability of lasers to produce brighter beams. The first time brightness was reported in relation to a laser beam was by Rampel in 1963, and showed that the obtainable power per unit area that could be produced for a diffraction limited beam is proportional to the brightness of a light source. Rampel further reported that such a laser beam is necessary for applications namely: microcircuit machining, drilling and cutting since controlled material removal in localized area was necessary. In the 1970 s and 1980 s papers were published by several authors in relation to increasing the laser beam brightness $[8,10]$, and the use of high brightness lasers in the military sector [11]. Thereafter, numerous papers were published with respect to enhancement of laser beam brightness and modification of the transverse mode [12], changing the cavity design within the optical resonator [16,22], and the beam delivery design [23,25]. However, only handful of publications could be found in relation to the effects of brightness, thereof on engineering and variety of general materials [26,35].

Last decade was said to be the decade of short-wavelength lasers by laser physicists [36]. Short wavelength lasers operate at ultra-fast pulse rates and are very much in demand in this decade but more so are the high brightness lasers namely the fibre laser in particular. The brightness of a laser beam is not frequently mentioned in laser processing research and or during general laser processing of materials in factory shop floor or a job-shop environment. Notwithstanding this, various investigators have shown the importance of these parameters and have demonstrated that considering brightness as a parameter could be useful, though, to a limited extent. Having said this, not only experimental investigations are called for to demonstrate the effect of laser beam brightness [26,35], but also an up-to-date literature review focused on various areas relating to brightness of laser beams is desired, as it has received very little attention over the years, since the introduction of a laser as a manufacturing tool.

\subsection{Significance of laser beam brightness}

High brightness lasers have been around since over a decade. Initially, it was the disc lasers, diode, tapered, semiconductor lasers and short-pulse, short wavelength excimer lasers that were in the industry, classified as high brightness sources but they suffered from lack of beam quality [37,42]. However, fibre lasers have been commercially introduced since 2005 [43]. They offer the missing aspects of the previous lasers. These are the superior beam quality along with many other technical advantages [43]. In recent years, a development of a solar pumped laser was also reported $[44,46]$. High brightness laser, however, offers considerable advantages over the conventional lasers. Those are, namely: superior beam quality; longer field of focus; smaller beam divergence; low cost per wattage; cleaner/sharper beam foot-print and higher power density than a low brightness laser. This allows cheaper laser process to take place, due to the usage of low cost per wattage. The high power density of a bright laser could also enable one to obtain higher processing speeds during laser material processing. High brightness fibre lasers could be considerably flexible, offering three dimensional processing of parts. Moreover, the high brightness also allows a tighter focus but a less expensive optics and beam delivery systems.

The reason why brightness is important in laser processing applications is because the intensity obtained within a focusing area through a lens is proportional to the brightness of the beam. High brightness laser processing allows fine spot size of the beam and creates a longer focusing distance. Hence, flexibility is further achieved with material processing as more distance is covered during laser operation. This is particularly offered by the fibre and the high power diode lasers (HPDL) and also the disc lasers [37,39].

High brightness laser sources such as a fibre laser or a HPDL produces high temperature during material interaction [36,47]. A fibre laser with high brightness in particular offers a longer depth of field (long focal length), small spot sizes, beam quality and a stable laser beam execution. The brightness of a laser is more effective in comparison to the laser power intensity or any other parameters singularly. This is because by achieving high brightness high processing temperatures would be generated [47]. The use of high brightness laser for material processing is also advantageous due to its potential for achieving low cost per wattage output [48].

The first reported work on laser beam brightness was in 1963 
1

$\begin{array}{ll}\text { Nomenclature } \\ \mathrm{Br} & \text { brightness } \\ \mathrm{D} & \text { density } \\ \mathrm{P} & \text { power } \\ P_{\mathrm{av}} & \text { average power } \\ A & \text { surface area of the beam cross-section } \\ \lambda & \text { laser beam wavelength } \\ M^{2} & \text { laser beam parameter product } \\ M_{X} \text { and } & M_{y} \text { average spacial dimensions of the beam } \\ S & \text { surface area } \\ I & \text { power per unit area } \\ \Omega & \text { solid angle of divergence }\end{array}$

by Rampel [7]. Having said that, brightness in relation to lasers became a concept in the 1970s. Earlier work in this field was somewhat focused on enhancing and improving the capability of lasers to produce brighter beams. Rampel showed that the obtainable power per unit area that could be produced for a diffraction limited beam is proportional to the brightness of a light source. Rampel further reported that such a laser beam is necessary for applications namely: microcircuit machining, drilling and cutting since controlled material removal in localized area is also necessary.

The work of Braid et al. [11] evaluated brightness as a unique parameter for the use of high energy lasers applied in the military sector. In particular, it was stated that the successful implementation of lasers as a weapon in the military sector would depend on its brightness. Braid et al. further went on to comment on the differences between an actual brightness and potential brightness. The suggested equation for the determination of potential brightness is shown in Eq. (5), and presented in Table 1. The difference between the actual brightness and the potential brightness is: at any point of the beam, the brightness could change due to the difference in beam diameter, the actual brightness maybe different to the potential brightness, since the potential brightness calculation does not take into account the losses [11]. This simply indicates that the actual brightness value of lasers would be more accurate but on the other hand, it is not always possible to understand and determine the losses of a laser system. This is why the potential brightness would be a good measure. A comparison could then be made with the actual brightness to reveal the losses.

\subsection{Research motivation and rationale}

This paper first provides a detailed review on the concept of radiometric brightness - the 'radiance'. This is particularly in relation to a unique light source such as a laser beam. Moreover, the review is also focused on how brightness as a concept is an important parameter to largely quantify a laser beam. Further, an update of current equations used for the determination of brightness was reviewed and proposed in a modified equation that provides a full characterization and optimization of laser beams by using its brightness as an important aspect. The paper then presents a to-date review of literature in relation to high brightness lasers to affect material processing and research studies previously conducted to modify laser system design to affect the laser beam brightness. This involves an update on the techniques used to improve the laser beam brightness and also extends the knowledge on how bright lasers were developed with enhanced/improved process capability which could allow cost reduction and improved efficiency with laser assisted processes in manufacturing. Lastly, a technique to characterize a laser beam by using the

$\begin{array}{ll}\Delta \Omega & \text { solid angle of subtended by the radiation } \\ \omega_{0} & \text { beam radius } \\ \theta & \text { divergence angle focus direction } \\ d & \text { diameter of the beam } \\ F & \text { focal length of focusing lens }(\mathrm{mm}) \\ \theta & \text { brightness (mrad) } \\ Q & \text { beam parameter product } \\ \omega \text { and } \theta & \text { emission width and full angle for lateral axis } \\ \text { BBP } & \text { beam parameter product } \\ n & \text { number of times diffraction limit } \\ I & \text { intensity } \\ \emptyset & \text { focus angle } \\ R & \text { radiance }\end{array}$

radiometric light property-radiance is rather encouraged. On account of this, one could consequently control the aspects relating to laser design, material processing energy usage and the associated cost in wattage. This paper is one-of-a-kind detailing the fundamentals of laser beam brightness as a concept and its importance in the laser processing, laser design and beam optimization areas. No such paper hitherto has shown studies in relation to any of the topics discussed herein with any depth. Moreover, the work in this paper could directly benefit larger/wider audience working in the aforementioned areas.

\section{Fundamentals of laser beam brightness with respect to previous work}

Brightness is somewhat a comparison of two rays of light which is generally judged by the human eye. This judgment is created from a variation in light intensities on the surface of the retina [1]. The general terminology of brightness of a light beam was classified in candles per square meter of light being emitted on a surface which was otherwise known as 'luminance' or 'radiance'. However, the application of the particular light source differentiates the use of either luminance or radiance $[2,48]$. As previously mentioned luminance is considered to be a photometric brightness and the radiance is the radiometric brightness [48]. Laser beams are monochromatic, unidirectional and coherent, which enable them to be focused in a small surface area, thus producing a brighter light when compared to other light sources. This also allows laser beams to exhibit very high power levels in a narrow spot size [1]. Brightness of lasers follows specific laws of optics $[2,49]$. Those are the following:

1. Brightness is an unchangable property and is not affected by focusing or defocusing a laser beam.

2. The brightness of lasers is generally not affected by any changes to the parameters by the end user $[20,21,29]$. But this is not true as a change in power, spot size, $M^{2}$, beam divergence and or the wavelength will lead to a change in the end brightness of the laser beam.

3. The brightness of a Gaussian beam does not change as it propagates. This is because the brightness is inversely proportional to the solid angle of divergence. The solid angle produced by a laser beam is proportional to the square of the divergence angle $\theta$. The smaller the divergence the higher the brightness of the laser. High brightness beams, however, have the most idealised beam profile and tend to have a high quality factor.

Laser beam parameters, namely; solid angle of divergence, wavelength, beam parameter product $\left(M^{2}\right)$, spot size and laser 


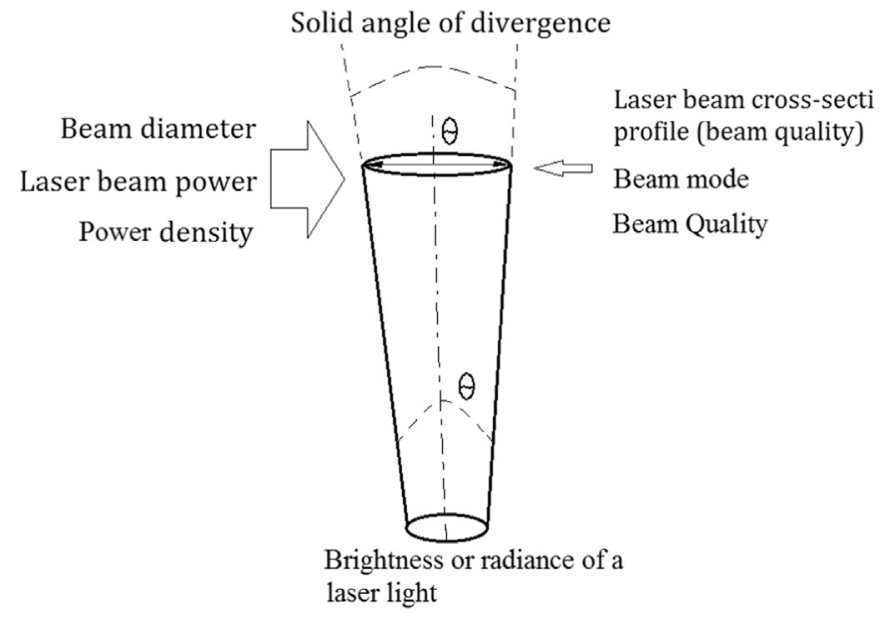

Fig. 1. A schematic diagram illustrating the parameters that equate to, and affect the radiance (brightness) of laser beams.

power are major contributors to the laser beam brightness and are used to calculate $[17,24,32,33,50,51]$ or to measure the brightness [31] and associated laser beam parameters [52,53]. However, practical measurement of the laser beam brightness is much complicated and involves time consuming set-ups. Thus, theoretical approach is more desirable and it could also be an accurate mean to determine the laser beam brightness.

The brightness in terms of a laser beam is calculated by the optical power divided by the product of the focused beam mode area $-M^{2}$, as well as the solid angle of divergence $(\Omega)$ as per the wavelength derived in Eqs. (1)-(4) [4,24,36,50,54,55]. Fig. 1 shows a schematic of the aspects of a laser beam that are taken into consideration when determining the laser beam brightness $(\mathrm{Br})$ [22].

$\mathrm{Br}=\frac{P_{\text {out }}}{A \Omega}$

where $P_{\text {out }}$ is the laser power over the surface area and $A \Omega$ is the solid angle of divergence. The solid angle of a Gaussian beam is shown by Eq. (2) [22]:

$\Omega=\pi \theta^{2}=\frac{\lambda^{2}}{w_{0}^{2}}$

where $\lambda$ is the wavelength and $w_{0}$ is the beam radius at the beam waste. For Gaussian laser beams that are circular, the propagation ratio is shown by Eq. (3).

$M^{4}=M^{2} \cdot M^{2}{ }_{\mathrm{x}}$

where $M^{2}{ }_{y}$ and $M_{x}^{2}$ are the parameters of the beam profile. Finally Eqs. (1)-(3), then result to Eq. (4) which is conventionally used to determine the brightness of the laser beam [22].

$\mathrm{Br}=\frac{P_{\text {out }}}{M^{4} \times \lambda^{2}}$

Eqs. (1)-(4) show the most commonly applied equation to determine the brightness. However, several authors have employed various equations as presented in Table 1 . This has led to a substantial amount of confusion as the equations are also not consistent to one-another. Thus, the work in Sections 3 and 4 of this paper elucidates the lack of clarity in previous work.

In particular, Eqs. (1)-(4) by Forbes et al. [22] do not take into account the laser beam spot size and only consider the use of output laser power. Eq. (5) by Braid et al. [11], does not account for the beam propagation factor both in $M_{x}$ and $M_{y}$ directions. Eqs.
(9)-(11), and (13)-(23), by several other researchers also do not take into account the spot size, whilst Eq. (12) considers the spot size and inherently, the laser power density, though, not the wavelength. Specifically, the full characteristics of a laser beam, namely: spot diameter and laser power (power density); $M^{2}\left(M_{x}\right.$ and $M_{y}$ ) and the directly proportional beam divergence; the wavelength of a laser beam were not previously considered in any of the equations. These characteristics equate to the brightness (radiance density) of the laser beam and are important in laser material processing. One can successfully determine the radiance density by fully considering the complete beam parameters to not only characterize the beam but also aid with process optimization.

\section{Relevant terminology in relation to laser beam brightness}

Literature in this area is somewhat confusing with respect to the terminology in relation to laser beam brightness. Luminance is defined as the direction of light emission which means that the brightness of an object is dependent on the direction or the angle from which the light is being visualized [3]. Luminance is also the intensity of light that is emitted from the surface. The intensity of light that is directed on a surface is classified as illuminance and is the opposite of luminance.

Particularly when working with laser beam brightness, it should be defined as radiance (power per unit area) in a solid angle of divergence (measured in steradian). On the other hand, misconception also occurs with the terminology 'irradiance'. This is the power per unit area (radiative flux) acting on a surface. The units of irradiance are: $\mathrm{W} / \mathrm{m}^{3}$, whereas, the referenced units of radiance in the literature is rather: $\mathrm{W} \mathrm{mm} \mathrm{sr}^{-1}$. It has been said that the use of units by previous workers is not consistent. Moreover, the aforementioned units only include the laser power $(\mathrm{W})$, spot diameter $(\mathrm{mm})$ and steradian $(\mathrm{sr})$. However, it is evident from the previous equations that the wavelength $(\lambda)$ would influence the end value of radiance considerably as demonstrated in our previous study [35].

Thus, we have proposed the following units: $\mathrm{W} \mathrm{mm}^{-2} \mathrm{sr}^{-1} \mu \mathrm{m}^{-2}$, as it was better suited, where laser power is (W), spot diameter is (mm), solid angle of beam divergence is (sr) and the wavelength is $(\mu \mathrm{m})$. The use of units in previous literature did not employ the laser wavelength $(\mu \mathrm{m})$, thus, the laser wavelength and its units $(\mu \mathrm{m})$ are included in our new proposed equation. Overall, radiance can be defined as the power from the source per area into a certain solid angle, and comparatively, the irradiance can be defined as the power onto a surface per area [33].

\section{Proposal of a modified equation: the radiance density equation}

Radiance in terms of laser light is not only a useful property, but is equally an important parameter as it is a means to measure how bright the laser beam is in radiometric terms. Measuring the brightness of a light source such as a laser would firstly help to characterize such a monochromatic, coherent, and unidirectional light. Secondly, it would aid to accurately measure the power intensity emitted by the light source by taking into account many different parameters that equate to radiance. Those parameters are namely: laser power; spot size (energy density); beam quality factor $M^{2}$; beam divergence and the wavelength.

The spot diameter, however, is not taken into consideration in previous studies and so, it would be essential to incorporate the spot diameter during the calculation of laser beam brightness. In order to do this, it is rather suitable to include the power density which is a combination of power and the spot size for a particular 


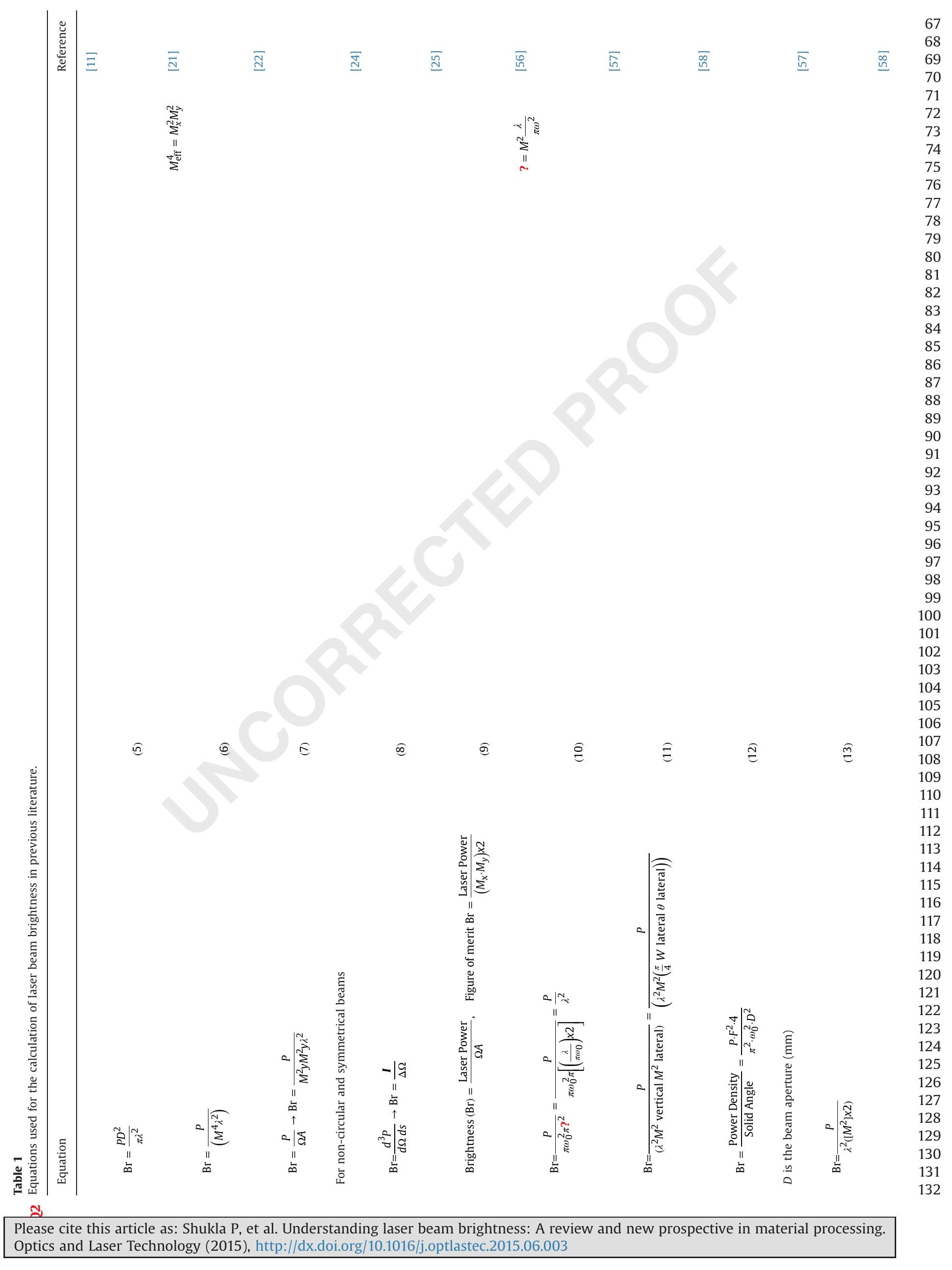




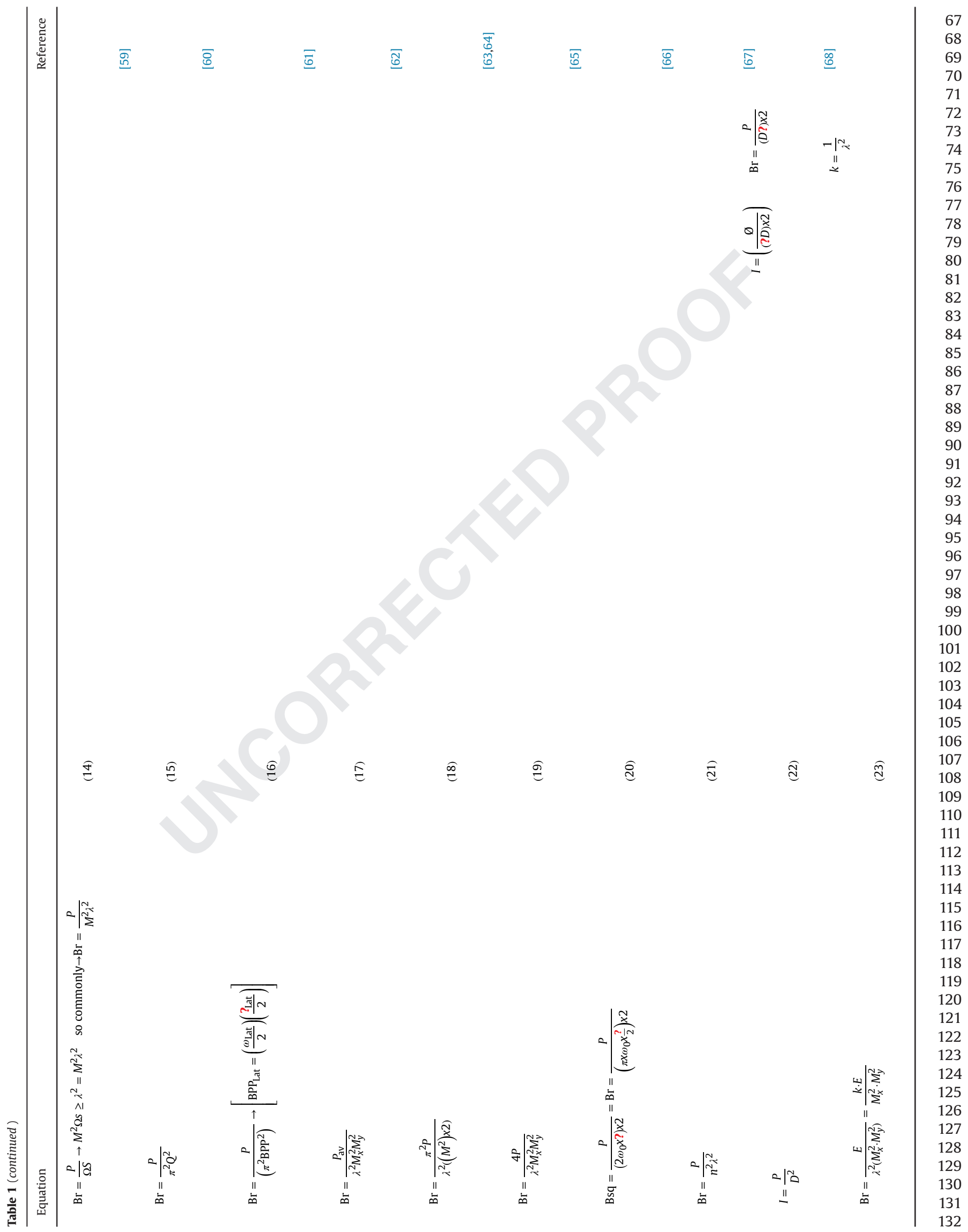


Gaussian or non-Gaussian beam. Equations presented herein showed that brightness is a function of output power, wavelength, $M^{2}$, the beam divergence and the spot diameter. Thus, to include the spot size, power density can be employed as an additional feature as derived in Eq. (24) for a 'top hat' (flat top) beam of an Nd:YAG laser used in this study as an example:

Power Density $=\frac{127}{d^{2}}$ xOutput Power $\left(\frac{\mathrm{W}}{\mathrm{cm}^{2}}\right)$

where $d$ is the laser beam diameter and 127 is for the top hat uniform beam profile with an equal energy distribution. In case the beam is Gaussian then the value 127 is replaced by 252 . Upon inclusion of the laser power density for determining radiance would in turn, give rise to Eq. (25). Eq. (25) adopts the use of power density rather than the conventionally used Eq. (4) or the equations presented in Table 1 . This in turn, would take into consideration the full laser beam parameters as mentioned previously and ultimately becomes the 'radiance density' (RD):

$\mathrm{RD}=\frac{(\mathrm{PD})}{M^{4} \lambda^{2}}$

where 'radiance $(\boldsymbol{R})$ ' replaces the conventional terminology of 'brightness (Br)', whilst, the 'radiance density (RD)' is used to take into consideration the beam diameter as well as the laser power (power density (PD)). If laser power is employed then radiance could be used but in case of full laser beam characterization, the above equation determining the radiance density (RD) would be rather suitable and accurate. $M^{4}$ is the cross-sectional laser-beam profile in the $x$ and $y$ directions $\left(M^{2}{ }_{y}\right.$ and $\left.M^{2}{ }_{x}\right)$. The beam profile $M^{2}$ is the laser beam propagation factor, inversely proportional to beam divergence. Hence, the beam divergence would be relative to the $M^{2}$ value. The laser beam wavelength $(\lambda)$ and the power density (PD) would account for the spot size and the input power of the laser beam. This equation is therefore, proposed for future studies, as it has more valid relationship using the correct terminology for radiance, since the power density is now included. Thus, all the parameters associated with calculating the radiance are now considered in our work. This will prove to be a complete method to characterize a laser beam of any kind as demonstrated.

\section{Previous endeavour in modification of laser beam brightness}

\subsection{Change in laser beam mode and the propagation factor}

Brightness or Radiance of lasers could be modified by various means. In particular, the parameters associated with the equations previously presented could be altered such that a slight modification could result to a considerable change of energy/at the nozzle and onto the surface of the work-piece. Specifically, it was the beam mode, quality, and beam divergence which were documented to improve the laser-beam brightness [69]. In addition, modification of the device design such as flared amplifiers, curvedand angled-grating cavities, and optical beam correction also assists in improving the performance of lasers through enhancing the brightness.

Hanna $[7,8]$ investigated the possibility of increasing brightness by changing the transverse mode. The first paper published by Hanna introduced brightness as a useful parameter, since it was reported that it is rather the brightness in comparison to the total emitted power that is required for materials processing. However, Hanna focused on the use of the transverse mode to alter the laser beam brightness by placing a plano-concave resonator adjacent to the plane mirror. This reduced the diffraction loss so that a $\mathrm{TEM}_{10}$ 
mode beam has the same diffraction loss as that of a $\mathrm{TEM}_{00}$ beam. This meant that a reduction in the divergence angle and $\mathrm{M}^{2}$ value, thereby, generated a higher laser beam brightness. This type of configuration produced a small size mode so average quality beams could be improved. Hanna concluded that this type of technique is yet questionable due to the lack of repeatability. Based on these studies it has shown that the laser beam mode is an important factor in altering the laser brightness. Changing the beam mode alters the beam quality and this also affects the beam divergence. The improvement of beam mode leads to a better $\mathrm{M}^{2}$ value and a smaller beam divergence angle. This characteristically enhances the laser brightness in the end. Changes in the $\mathrm{M}^{2}$ value is another way to enhance the brightness, but the beam quality, transmissive electro-magnetic (TEM) mode, as well as a highlydiffused cross-axis beam delivery cavity (HiDiCAB) could be used to enhance the brightness of a Nd:YAG laser as demonstrated by Hirano [70] and Abdolvand et al. [71]. Yanson et al. [72], demonstrated an increase in brightness of a high power diode laser by reducing its far-field divergence. This improved the beam profile and led to obtain a superior beam propagation factor. This in turn, increased the brightness with the use of a epitaxial design to generate the laser beam. Machavariani et al. [73] employed a mode converter which radially polarized a beam to improve the beam quality from $M^{2}=2.5$ to $M^{2}=1.3$. This gave rise to the output brightness of the laser by a factor of 2.5 [73].

\subsection{Modification of optical resonator, beam delivery and optics}

Varnavsky et al. [10] conducted a similar investigation by using an unstable resonator design. This comprised of a concave and convex mirror assembly. This allowed a tighter beam divergence. Consequently, it created a laser beam close to its diffraction limit with a mode of $\mathrm{TEM}_{00}$ and to characteristically exhibit a high brightness laser.

Marshall et al. [16] increased the brightness of a laser by modifying the diode design. This changed the $M^{2}$ from one direction and produced a better beam quality. This idea was somewhat close to that of Hanna. However, the approach was by slicing the diode beam in a grating prism as fabricated by optic extrusion technique which allowed a low-cost manufacture. Monjardin et al. [23] also modified a laser beam quality by using correction optics and improved the beam pointing accuracy and its quality. This in turn, allowed an uncorrected beam of $\left(M^{2}=12.26\right)$ to reduce to $M^{2}=2.33$, allowing a tighter focus.

Machavariani et al. [12,13] also enhanced the brightness of a high power laser by changing the beam mode from a lower order LG $(0,1)^{*}$ beam to a near enough Gaussian beam with a $M^{2}$ of 1.034 from a previously known theoretical value of $M^{2}=2$, at laser power of $30 \mathrm{~W}$. Machavariani adopted two techniques to achieve the same results. The first technique was to radially polarize the mode into disintegrated modes with orthogonal polarization. Then one of the modes were rotated whilst both disintegrated modes were flattened. Thereafter, the modes were combined coherently on a non-polarizing beam splitter to almost achieve a doughnutshaped beam. The features of the doughtnut shaped beam comprised of a bright central peak and a ring as its end profile. In Machavariani et al.'s second mode modification technique, the radially polarized mode was polarized to disintegrated, flattened modes and were then combined incoherently on a polarizing beam splitter. This in turn, resulted in a bright central peak mode comprising of four side lobes at its end profile with at least $20 \%$ power $[15,16]$. The beam quality factor as a result of the mode change was improved from $M^{2}=2.52$ to $M^{2}=1.3$ at a laser power of $30 \mathrm{~W}$. Thus, the brightness was enhanced by a factor of 2.5 . 3 Brown et al. [26] introduced a technique for obtaining a linearly polarized transverse electric field output which when combined with a diode-bar pumped planar waveguide offers a route to a high-power polarized planar waveguide laser. This consequently improved the $M^{2}$ and the brightness of the laser.

A new fibre coupled diode laser was designed with a high brightness and was said to be cost effective to manufacture and was costing less than $\$ 20 /$ watts. An output power $500 \mathrm{~W}$ was directed through a $200 \mu \mathrm{m}$ fibre diameter at $976 \mathrm{~nm}$ wavelength. This was done by a specially tailored laser bar (T-bar) with special collimator lenses. This coupled the beam into a $200 \mu \mathrm{m}$ fibre diameter. Multi-application of T-bar at identical wavelength resulted in emitting high power level of up to $1 \mathrm{~kW}$ [27].

Zhao et al. [14], developed a new technique to transform a high order beam mode $\left(\mathrm{LP}_{11}\right)$ into a near Gaussian profile with an enhanced laser beam propagation factor. Different lobes were modulated in Zhao et al.'s work to increase the laser power by a factor of 1.5 in the far field range. This showed tighter beam divergence angle, increased beam propagation factor leading to enhanced brightness value. Moreover, Shalaby et al. [15] also developed a new method to control the beam mode. This would emit a uniform and a bright laser beam. A particular ring cavity was constructed with an ammolifying multicore (19-core, Yb-laser) to generate a diffraction limited beam output. This accordingly, resulted to an improved laser beam brightness by enhancing the $M^{2}$ to 1.21 .

Forbes et al. [22], modelled the brightness from a cross porroprism resonators and showed that the brightness is significantly influenced by the angle between the two prism edges. At higher prism angles the brightness was increased as fewer petals of the beam foot-prints were to be seen, with decreasing angles and increasing the number of petals of the beam foot-print showed decreasing the prism angles, and inherently a reduction in the brightness.

Li et al. [17] worked on the reliability and efficiency of high brightness lasers by using the $940 \mathrm{~nm}$ wavelength to show the maximum power conversion efficiency of $60 \%$ at an output power of $72 \mathrm{~W}$ with a very good beam quality. Treusch et al. [18] applied a high-brightness semiconductor laser and found that collimation lenses can be used to increase the brightness of the laser by a factor of two. In addition, the wavelength and polarization coupling also contributed in increasing the end brightness. Leibreich and Treusch [19] conducted a similar investigation on improving the brightness of a semiconductor diode laser using laser beams of various wavelengths to increase the output power and the brightness. The results showed that laser-beam brightness can be doubled without any changes to the $M^{2}$ value, unlike other published literature. This in general could be a new prospective within the laser materials processing industry. However, altering the brightness involves careful measurement of the laser beam and is considerably difficult to measure for an industrial laser as it requires complex set-up and a time consuming procedure.

High brightness lasers sources such as disc, fibre, and diode lasers are conventionally popular but diode lasers generally lack the power and the beam quality to exhibit high power density [74]. Having said that, micro-optics have been developed in recent years to improve the output of the high power diode laser (HPDL), because the beam executed by such a laser source was in multiple rays and does not point in the same direction. This makes the laser somewhat deficient and decreases the employability for the high demanding application laser such as a HPDL. It was cited that not just the HPDL but other laser source also could benefit from an increased brightness so that efficiency could be improved in areas where there is an extensive use of lasers. In order to do this, custom made lenses (micro-optics) were designed to enhance brightness of laser beams as reported by McBride [23]. The microoptics were as such that they provide a correction to deficiency of each laser source at individual level. Results showed, enhanced 


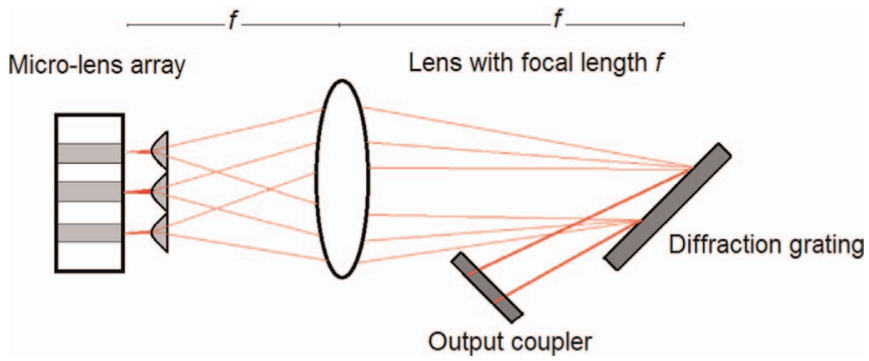

Fig. 2. A schematics of wavelength beam-combining laser diode arrays to enhance brightness [23].

brightness with improved efficiency of $80 \%$ compared to previously developed lasers that were only $15 \%$ efficient [23].

Multiple laser beam combining technique has also been reported to have influenced the brightness of laser beams [75]. The results showed that six-mirror cavity lasers could generate a brightness value of $12.66 \mathrm{~mJ} / \mathrm{m}^{2}$ St in comparison to less cavities present within the system. Brightness was also cited to have increased by combining wavelength laser beam diodes [20]. Using micro-lense arrays, 6 diode laser arrays were passed through a lens at a certain focusing length onto a diffraction grating. This directed the beam into an output coupler and onto the processing material as shown in Fig. 2 [23]. In this wavelength, beam combining of diode $(\mathrm{WBC})$ lasers generated arrays of small light rays that were focused to a much intense and brighter beam that in other cases required high power laser systems which consequently were also not so cost effective. This type of design was configured using a diode laser which was claimed to be the first in cutting and welding of metallic materials of thickness 0.26 in. and led to a reduction in cost, increase in efficiency in comparison with diode pumped, fibre and solid state lasers.

Another investigation involved the use of HPDL operating in both the near- and mid-infra-red regions. The laser resonator design was modified comprising of certain stripe that had an etched semiconductor layer [22]. This would also limit the electrical current injection into the diode laser. The diode laser stripe has variation in width in the lateral direction which in turn controls the output power. This in other words means that broad area $(150 \mu \mathrm{m})$ given for the laser beam allowed more waste to exhibit at the output end and allowed $\times 3$ increase in brightness $\left(100 \mathrm{MW} / \mathrm{cm}^{3}\right)$ to be generated in particular with the near infrared (NIR) wavelength. However, broad cavity concept used for the NIR wavelength $(800-1000 \mathrm{~nm})$ is not very practical with the midinfra-red (MID) wavelength lasers and therefore, Kelemen et al. [58] proposed an alternative resonator design which involved the use of a tapered laser resonator. This was reported to avoid the limitations of the previous design suited for the NIR wavelength lasers. This type of design also allows increase in brightness for lasers with wavelength ranging from 1800 to $2500 \mathrm{~nm}$.

Wang [76] developed a laser beam shaping technique which enhanced the brightness of a diode laser up to $2.4 \mathrm{MW} / \mathrm{cm}^{2} \mathrm{sr}$. The technique was such that the laser diode array was re-shaped using groups of prisms which created a beam with a so called 'smiling' foot-prints. This upon directing through an optical fibre of $0.6 \mathrm{~mm}$ diameter gave an ultimate brightness value of $8 \times 10^{4} \mathrm{~W} / \mathrm{cm}^{2} \mathrm{sr}$.

Bergmann et al. [77] reported that using lasers with efficiency up to $45 \%$, the beam parameter product still has to be high, as well as the focusing ability of the system. Both combined leads to a high brightness beam which is important for applications such as laser spot welding [77]. A beam combining technique was used to improve the brightness of a fibre laser by Sprangle [78]. This used an array of three fibres focused at a $45^{\circ}$ mirror which was then focused at the target. It improved the efficiency, and produced a compact, robust, low maintenance, high energy and long lasting laser system. Results showed propagation efficiency of $90 \%$ at a range of $1.2 \mathrm{~km}$, at $3 \mathrm{~kW}$ laser power in moderate turbulence within the atmosphere [78]. Ishizuka et al. [79] stated that the brightness of field-emission tips (electron microbeams) could be substantially improved by using a magnetic field instead of the electrostatic lens to suppress the divergence of such microbeams which inherently enhanced the actual brightness. This concept could be adopted to lasers in general, whereby the wavelength as such can be extended to function in the X-ray wavelength regions at considerable energy densities.

Almeida and Liang [44,45] designed a high brightness solar pumped laser in which a maximum brightness of $9 \times 10^{-1} \mathrm{~W}$ was obtained at a beam mode of $\mathrm{TEM}_{00}$. A $30 \%$ increase in brightness was reported. This was achieved by the adoption of compact fused silica light-guide assembly and the use of a core-doped ceramic rod with a $5 \mathrm{Mm}$ diameter and Sm:YAG cladding that proved to be a brightness enhancer for a solar-pumped laser.

Ronen et al. [80] designed a new compact slab laser configuration comprising of a passive combining of several laser channels. In this configuration, the beam is polarized and transferred on to an adjacent channel by using a polarizing beam-displacer which aids in obtaining a combined output of higher brightness [80]. Enhancement in brightness of electron beams was also reported by few other authors, namely: Bluem et al. [81] and Zolotrev et al. [82]. Bluem et al. developed a high brightness electron gun which could be utilized for a variety of applications such as superconducting CW RF and a DC/SRF gun which prove to be good sources for light injectors. Zolotrev et al. also mentioned that such high brightness devices are useful for electron microscopy, inverse photo-emission, low energy electron scattering and electron holography.

\section{State-of-the-art in high brightness laser-material processing}

Several workers in recent time have worked with high brightness lasers for processing materials $[20,21,28-36,48,50,54,56,58]$. Wallace [20] used the high brightness diode laser to produced high efficiencies and lower operating costs. Wenzel et al. [21] used a high brightness semiconductor laser to show that high reliability and efficiency can be achieved with a low beam quality factor. Brown and Frye [29] worked with high brightness cutting and drilling process for aerospace materials with the aid of a Nd:YAG laser. The results showed improved cutting and achieved shallow angle holes.

Investigation conducted by Del Val et al. [30] showed the effects of laser cladding of stainless steel plates and co-based superalloy (powder coating material) using a Nd:YAG laser and a $\mathrm{Yb}$ : YAG fibre laser. The findings revealed that fibre laser in comparison to that of the Nd:YAG showed versatile parameter window and enlarged clad track and deeper penetration, but similar hardness was obtained from applying both the lasers. Val et al. then reported that this effect had occurred because of the higher beam quality as well as the high brightness produced by the fibre laser. This generally is ideal for creating thinner clad tracks but a Nd:YAG laser is ideal for producing wider clad tracks. Previous investigations conducted by the authors of this paper $[30,33]$ on the the effects of brightness between the Nd:YAG laser and the fibre laser on engineering ceramics, namely: $\mathrm{ZrO}_{2}$ and $\mathrm{Si}_{3} \mathrm{~N}_{4}$ and compared brightness effects on ceramics of two lasers, using identical process parameters and similar wavelength, but with different beam brightnesses. Results showed that the ceramics were more affected by the high brightness fibre laser compared to a low brightness Nd:YAG lasers, and produced difference in hardness, track widths and the ceramic microstructure.

Verhaeghe and Hilton [60], investigated the welding performance on $5 \mathrm{~mm}$ and $10 \mathrm{~mm}$ thick S275 type C-Mn steel and 5083O type aluminium alloy using several lasers with $1064 \mathrm{~nm}$ 
wavelength, namely: Nd:YAG; Yb:Fibre; Yb:Fibre and Yb:YAG disc lasers. The use of brightness as a beam property was also encouraged in this paper, and it was reported that the $\mathrm{M}^{2}$, and spot size increases the laser beam brightness, though, to what extent was not reported. However, the depth of penetration after conducting the welding trials on both the steel and aluminium showed that the high brightness fibre laser penetrated deeper into the material. Verhaeghe and Hilton also mentioned that an optimal brightness between 32 and $38 \times 10^{5} \mathrm{~W} / \mathrm{mm}^{2}$ steradian ( $\mathrm{sr}$ ) would be ideal for aluminium and steel [60]. The value obtained for brightness based on the parameters used for the welding experiments compared to the brightness equations employed in previous research differed. This indicated that there was no generic equation employed for determining the brightness, thus, discrepancies in the results of various published literature were found.

A high brightness fibre laser was reported to have a high efficiency ratio during a laser spot welding process of an aluminium as reported by Bergmann et al. [77]. This was due to the $M^{2}$ being lower. This in turn, resulted to the high brightness fibre laser and disc lasers to produce the highest wall plug efficiency compared to other lasers. The typical wall plug efficiency found for the fibre laser was $25-30 \%$ at a $M^{2}$ of 2 and the same for the disc laser. This was the highest in comparison to other laser sources employed. Having said that, no brightness values were given in relation to the parameters employed for the laser spot welding process in that study [77].

One other application where high brightness lasers could be useful is in laser assisted ignition. Shaw et al. [83] reported that fast ignition can be achieved with the use of a high brightness $\mathrm{KrF}$ laser system with a low wavelength $(268 \mathrm{~nm})$. The paper also reported that femtosecond pulses could obtain a significantly high brightness value and this is rightly so due to the low wavelength of the KrF laser which could generally exhibit a high brightness value. An interesting aspect for future study is the relationship of short and ultra-short pulse lasers with its beam brightness. It can be predicted from the investigation of Shaw et al. that fast pulses (400 fs) produced a sufficiently high brightness in comparison to $60 \mathrm{ps}$, but to establish this further, a broader and wider investigation is required for further understanding.

\section{Laser beam brightness as a parameter in materials proces- sing: outlook and new research prospective}

Having undergone a wider, extensive literature review in this area to-date (2015), we propose that brightness as a parameter of a laser beam should be given importance in the laser processing industry. This is because an intensively bright laser beam allows high power per unit area through the optics, nozzle and consequently at the workpiece. This makes an immense difference to the material being processed. In any case, not just material processing, but fibre and solid state laser pumping; remote sensing; medical industry (dentistry, ophthalmology); imaging; printing; military; entertainment industry, and lasers applied in space research may also benefit. A high brightness laser could minimize the optical complexity, as well as packaging requirements and help to deliver the photon energy with minimal losses and at higher efficiency.

Furthermore, for measuring the brightness of distant stars and planets in space research, the general theory is that the more shiny the object (star/planets), the higher its brightness, temperature, and the size in relation to the distance from which it is observed. In addition, the bright object in space also glows at high temperature, which in turn makes it a hot star and the planet equivalently glows with bigger reflection which enables further understanding of a star as well as the planets' composition using its brightness [84,86]. Likewise, brightness of lasers could relate to the same, since the shinier the laser material interaction, the brighter and hotter the plume glows and therefore, may result to high temperature and inherently is postulated to exhibit a high temperature laser material interaction. With precise control of parameters, the temperatures at the interaction zone could also be varied resulting in desirable phase changes to the material and low usage of energy. Brightness of lasers is not visible from a naked eye particularly if they are in the infra-red region and therefore, the shine or the glow as previously mentioned is only visible via an infra-red wavelength measuring device such as a pyrometer or an infra-red temperature measurement thermometer. It would also be ideal to understand the brightness temperature of various lasers at different wavelengths. For instance, Doubenskaia et al. [36] conducted an investigation which measured the laser material interaction zone during a cladding process. This in turn was simply used to determine the heat map using calibration with a black body. This was however, for a continuous wave (CW) Nd:YAG laser, but for future studies, it would be valuable to take into consideration the various lasers, from variety of wavelengths with beam parameters set constant, and the traverse speed would enable one to understand the effects of different brightness lasers with respect to the processing temperatures which it emits. This will contribute further to ones understanding of the effects that brightness has upon the material despite applying identical laser parameters.

Brightness measurement of a laser is a difficult practice and requires a complicated procedure. The correct measuring technique for brightness of a laser beam is not strategically documented anywhere except the use of the referenced equations presented herein but measurement of beam divergence and the $M^{2}$ value is documented in the British standards [52,53]. Measuring the two enables the calculation of brightness by placing values of beam divergence, and the $M^{2}$ value into the equation.

Upon reviewing the available equation for the determination of laser beam radiance, it was clear that the parameters that can affect brightness are the laser power; spot diameter and combining those would be the power density; $M^{2}$ value and the inversely proportional solid angle of beam divergence and the laser beam mode. These parameters of the laser beam to some extent-or-another, all contribute to the modification of laser radiance. However, it is not clear to what extent and therefore, an independent study is currently being undertaken which is focused on the contribution of such parameters to demonstrate a deeper understanding of this topic.

\section{Conclusions}

Firstly, this review paper addresses the correct terminology for brightness. This is 'radiance' for a laser beam. This is so because a laser beam is a unidirectional, monochromatic and coherent ray of light and is rather classified as a radiometric light. This paper reviewed existing empirical equations previously employed to determine the brightness (radiance) and presented a modified equation that was more applicable to determine the radiance of lasers. In addition, we have also shown a review of different techniques adopted by previous investigators to improve the brightness of laser beams. The laser beam brightness can be enhanced by several means such as changing or improving the $M^{2}$, and beam mode correction using better optical arrangements to give sharper beam quality, leading to $M^{2}$ value being closer to the diffraction limit. Both consequently provide a smaller solid angle of divergence, whereby, radiance of the beam is increased.

What is more, to-date literature was reviewed regarding the 
high brightness laser processing of materials and showed that a gap in knowledge exists in relation to the laser material interaction with bright lasers, and the individual cost per wattage all need to be addressed in future studies.

The use of brightness or radiance as a concept in laser processing practices is a perfect mean to monitor, characterize and carry-out laser process optimization and process refinement. This is because of the inclusion of all related laser beam parameters within the brightness equation. Therefore, a future investigation would be focused on the individual contribution of each of these parameters to effect radiance by demonstrating the relationship of each parameter with one-another, namely: laser power; spot size (power density); $\mathrm{M}^{2}$, beam divergence and the wavelength to effect the end value of radiance. Doing so would enable the improvement of laser design, laser operations such as, sheet-metal cutting; precision mass production marking; large area engraving; laser welding; deep drilling; laser peening and surface treatment in general, and many other laser applications in manufacturing/ production environment where lasers are applied.

\section{Competing interests}

We have no competing interests.

\section{Funding}

None of the authors were funded to conduct this research, thus, this research is not funded by any individual or organization.

\section{Uncited references}

$$
[9,38,40,41,85] \text {. }
$$

\section{Acknowledgements}

The authors of this paper would like to confirm that this research is not associated with any conflict of interest, and is also not in any financial ties with individual or any organization.

\section{References}

[1] Breck HC. Understanding of laser Technology. Tulsa: Penn Well Publishing; 1991.

[2] Milonni PW, Eberly JH. Lasers. New York: John Wiley \& Sons; 1998.

[3] Robieux J. High Power Laser Interaction. Paris: Lavoisier Publishing; 2000.

[4] Paschotta R. Encyclopaedia of Laser Physics and Technology. Berlin: Wiley$\mathrm{VCH} ; 2008$.

[5] Reesinck JJM. Distribution of brightness and colour over the sky. Physica XII 1946;5:296-300.

[6] Zhang W, Lu D, Chang Y. Feasibility study of cloud base height remote sensing by ground-based sky thermal infra-red brightness temperature measurements. Proc. SPIE 2010;5832:228-43.

[7] Rampel RC. Appl. Phys. Laser Tech. Bull. 1963;1963(1).

[8] Hanna DC. Increasing laser brightness by transverse mode selection-1. Opt. Laser Technol. 1970:122-5.

[9] Hanna DC. Increasing laser brightness by transverse mode selection-2. Opt. Laser Technol. 1970:175-8.

[10] Varnavsky OP, Kirkin AN, Leontovich AM, Mirzoyan RG, Mozharovsky AM, Solomatin IJ. Passively mode-locked Nd:YAG and ruby lasers with high brightness per unit active volume. Opt. Commun. 1983;45(5):342-5.

[11] Braid TH, Volpi A De, Herzenberg CL, Ringo GR, Stanford GS. Laser Brightness verification, Science \& Global Security. . SA: Gordon and Breach Science Publishers; 1990. p. 59-78.

[12] G. Machavariani, N. Davidson, Y. Lumer, I. Moshe, A. Meir, S. Jackel, New methods of mode conversion and brightness enhancement in high-power lasers, in: Proceedings of the Lasers and Electro-optics and the International
Quantum Electronics conference, Munich, 17-22 June, 2007, p. 1. http://dx.doi. org/10.1109/CLEOE-IQEC.2007.4385831.

[13] Machavariani G, Davidson N, Lumer Y, Moshe I, Meir A, Jackel S. New methods of mode conversion and beam brightness enhancement in a high-power laser. Opt. Mater. 2008;30(11):1723-30.

[14] Zhao H, Wang X, Ma H, Zhou P, Ma Y, Xu X, Zhao Y. Adaptive conversion of a high-order mode beam in top a near-diffraction-limited beam. Appl. Opt. 2011;50:4389-92.

[15] Shalaby BM, Kermene V, Pagnoux D. 19-Cores Yb-fibre laser with mode selection for improved beam brightness. Appl. Phys. B: Lasers Opt. 2009;100 (4):859-64.

[16] M.L. Marshall, R.F.K. Kleine, J.B. Renton, G.D. Matthews, R. Bhatia, Light slicer for increasing diode brightness, CLEO 2000, CTh M69, 2000, p. 498

[17] Li H-X, Truchan T, Brown, Pryor R, Pandey R, Reinhardt F, Mott J, Macomber SS. Reliable high-efficiency high-brightness laser diode bars at $940 \mathrm{~nm}$. Opt. Laser Technol. 2004:36(4):327-9.

[18] Treusch HG, Ovtchinnikov A, He X, Kanskar M, Mott J, Yang S. High-brightness semiconductor laser sources for materials processing: stacking, beam shaping, and bars. IEEE J. Sel. Top. Quantum Electron. 2000;6(4):601-14.

[19] Leibreich F, Treusch HG. Innovative stacking techniques increase the output power and brightness of diode laser bars for materials-processing applications. Micro/Nano Lithography \& Fabrication. SPIE Newsroom; http://dx.doi. org/10.1117/2.5200109.0004.

[20] Wallace J. Direct-diode lasers combine to form powerful, high-brightness beam. Laser Focus Word 2009;2009:24-5 (June Issue).

[21] Wenzel H, Bernd S, Erbert G. High brightness diode lasers. C. R. Phys. 2003;2003(4):649-61.

[22] Forbes A, Burge L, Anatolievich L. Modelling laser brightness from cross Porro prism resonators. Laser beam shaping VII. Proc. SPIE 2006;6290(62900):M28.

[23] R. McBride, 'Micro-optics' make laser beams brighter, An R\&D case study, Technology Strategy Board, Driving Innovation, Innovation Results, T12/029 2012, 2 p., 〈www.innovateuk.org .

[24] J.F. Monjardin, K.M. Nawak, A.R. Holdsworth, H.J. Baker, D.R. Hall, Brightness improvement for micro-lensed, laser diode bar stack Lasers \& Electro-Optics Europe, CLEO/Europe, 22-27, June 2003, p. 251.

[25] Shepelev V. About transforming of radiation brightness in optical process. Am. Assoc. Phys. Technol. 2009;78(2):158-9.

[26] Almeida J, Liang D, Guillot E. Improvement in solar-pumped Nd:YAG laser beam brightness. Optics Laser Technol. 2012;44:2115-9.

[27] C.T.A. Brown, R.D. Harris, D.P. Shepherd, A.C. Tropper, Member, IEEE, J.S. Wilkinson, B. Ferrand, A Polarized Brightness-Enhanced $\mathrm{Nd}: \mathrm{Y}_{3} \mathrm{Al}_{5} \mathrm{O}_{12}$ Planar Waveguide Laser, 1998.

[28] M. Haag, B. Köhler, J. Biesenbach, T. Brand, Novel high-brightness fibre coupled diode laser device, in: Proceedings of SPIE 6456, High-Power Diode Laser Technology and Applications, V 64560T, 2007.

[29] Brown RT, Frye RW. High-brightness laser cutting \& drilling of aerospace materials. Proc. ICALEO-1996 Sect. C 1996:78-85

[30] Del Val J, Comesaña R, Lusquiños F, Boutinguiza M, Riveiro A, Quintero F, Pou J. Laser cladding of Co-based superalloy coatings: comparative study between Nd:YAG laser and fibre laser. J. Surf. Coat. Technol. 2010;204:1957-61.

[31] Shukla P. Viability and Characterization of laser surface treatment of engineering ceramics (A doctoral thesis). Loughborough University; 2011.

[32] Shukla PP, Lawrence J, Paul A. Influence of laser beam brightness during laser surface treatment of $\mathrm{ZrO}_{2}$ engineering ceramics. Lasers Eng. 2012;22(34):151-73.

[33] Shukla PP, Lawrence J. The influence of brightness whilst laser surface processing of a silicon nitride engineering ceramic. Opt. Lasers Eng. 2012;50:1746-51.

[34] Shukla PP, Lawrence J. Role of laser beam radiance in different ceramic processing: a two wavelength comparison. Opt. Laser Technol. 2013;54:380-8.

[35] Shukla PP, Lawrence J. Identification of Optical Parameters for Determination of Radiance. J. Opt. Opt. Soc. India 2015;44(1):12-9.

[36] Doubenskaia M, Pavlov M, Grigoriev S, Smurov I. Definition of brightness temperature and restoration of true temperature in laser cladding using infrared camera. Surf. Coat. Technol. 2013;220:244-7.

[37] Sumf B, Hülsewede R, Erbert G, Dzionk C, Fricke J, Knauer A, Pittroff W, Ressel P, Sebastial J, Tränkle G. High brightness $735 \mathrm{~nm}$ tapered lasers-optimisation of the laser geometry. Opt. Quantum Electron. 2003;35:521-32.

[38] A. Otani, A High-Brightness Nd:YAG Laser Processing Machine, Technical Reports Mitsubishi Electric Advance 1997, pp. 22-24.

[39] Rudy P, Steele T, Vaissie L. More efficient and less complex-enhancing the spectral and spatial brightness of diode lasers, lasers technology, laser beam source. Laser+ Photonics 2008:32-6.

[40] S.V. Govorkov, Sol P. DiJaili, D.W. Anthon, L.A. Spinelli, High Power and High Brightness Diode-Laser Array for Material Processing Applications, United States Patent, Patent No. US 7697207B2, 2010, 2 p.

[41] M.L. Osowski, W. Hu, R.M. Lammert, T. Liu, Y. Ma, S.W. Oh, C. Panja, P.T., Rudy, T. Stakelon, J.E., Unger, High brightness semiconductor lasers, QPC Lasers, Inc., in: Proceedings of 2007 SPIE Photonics West Paper: 22nd Jan 2007, LASE Conference 6456, Session 3, 7 p.

[42] Glas P, Naumann M, Schirrmacher A. A novel design for a high brightness diode pumped fiber laser source. Opt. Commun. 1996;122:163-8.

[43] O'Nei W, an K. High-quality micromachining of silicon at $1064 \mathrm{~nm}$, using a high-brightness MOPA-based 20-W Yb fiber laser. IEEE J. Sel. Top. Quantum Electron. 2009;15(2):462-70. 
[44] Almeida J, Liang D. Design if a high brightness solar-pumped laser by lightguides. Opt. Commun. 2012;285:5327-33.

[45] Liang D, Almeida J. Design of ultrahigh brightness solar-pumped disk laser. Appl. Opt. 2012;15(26):6382-8.

[46] Richardson DJ, Nilsson J, Clarkson A. High power fibre laser: current status and future perspectives. J. Opt. Soc. Am. 2010;27(11):B63-91.

[47] Chalupsky J, Bohacek, Hajkova P, Hajkova V, Hau-Riege SP, Heimann PA, Juha L, Krzywinski J, Messerschmidt M, Moeller SP, Nagler B, Rowen M, Schlotter WF, Swiggers ML, Turner JJ. Comparing different approaches to characterization of focused X-ray laser beams. Nucl. Instrum. Methods Phys. Res. A 2011;631:1303.

[48] Dieh R. High Power Diode Laser Fundamentals, Technology, Application. Berlin, Germany: Springer Verlag; 2000.

[49] Das P. Laser and Optical Engineering. New York: Springer Verlag; 1991.

[50] Rudiger P. Encyclopedia of Laser Physics and technology. Berlin: Wiley-VCH; 2007.

[51] Ready FJ. Industrial Applications of Lasers. New York: Academic Press Inc.; 1978.

[52] Ion JC. Laser Processing of Engineering Materials. Oxford, UK: Elsevier Butterworth Heinemann; 2005.

[53] Koechner W. Solid-State Laser Engineering, 5th Revised and Updated Edition. Berlin, Germany: Springer-Verlag; 1999.

[54] British Standards, Las0065rs and Laser-related Equipment-Test Methods for Laser Beam Widths, Divergence Angles and Beam Propagation Ratios-Part 1: Stigmatic and Simple Astigmatic Beams, BS EN ISO 11146-1, 2005.

[55] British Standards, Lasers and Laser-related Equipment-Test Methods for Laser Beam Widths, Divergence Angles and Beam Propagation Ratios-Part 2: General Astigmatic Beams, BS EN ISO 11146-2, 2005.

[56] SPIE, Brightness or Radiance of Laser Beams, Laser Beams, The Society of Photo-Optical Instrumentation Engineers, 2008, p. 21 〈http://ebooks.spiedigi tallibrary.org/content.aspx?bookid=106\&sectionid =31558267). http://dx.doi. org/10.1117/3.767474.p21.

[57] Encyclopedia of Laser Physics and Technology, 〈www.rp-photonics.com/ brightness.html>.

[58] Kelemen K, Gilly J, Friedmann P. High-brightness near-and-mid-infrared diode lasers. Photonics Int.: Laser Sources 2010:8-9.

[59] A. Kudryashov, H. Weber (Eds.), High-Brightness Solid State Laser Systems with Phase-Conjugating Mirrors, Laser Resonators: Novel Design and Development, 1999, pp. 97-117.

[60] G. Verhaeghe, P. Hilton, 2005, The Effect of spot size and laser beam quality on welding performance when using high-power continuous wave solid-state lasers, in: Proceedings of 24th International Congress on Applications of Laser \& Electro-Optics, October 31-November 4, 2005, Miami, FL, USA.

[61] Jeff Hecht, Photonics Frontiers: High-Power Laser Diodes: Bringing High Brightness to High-Power Laser Diodes, Laser Focus World, Summer Issue 2011, 〈http://www.laserfocusworld.com/articles/print/volume-47/issue-11/fea tures/bringing-high-brightness-to-high-power-laser-diodes.html/.

[62] Vijayakumar D, Jenson Bjarlin O, Thestrup B. 980nm high brightness external cavity broad area diode laser bar. Opt. Express 2009;17(07):5684-90.

[63] Denis Saint, de R, Nocolas Passilly, Ait-Ameur K. Laser Beam brightness of Aperture optical resonators. Opt. Commun. 2006;264:193-202.

[64] Kemp AJ, Valentine JG, Burns D. Progress towards high-power, high-brightness neodymium-based thin-disk lasers. Prog. Quantum 2004;28:305-44.

[65] Sumpf B, Hasler Karl-Heinz, Adamiec P, Bugge F, Dittmar F, Fricke J, Wenzel H, Zorn M, Erbert G, Tränkle G. High-brightness quantum well tapered lasers. IEEE J. Sel. Top. Quantum Electron. 2009;15(3):1009-20.

[66] Wilson J, Hawkes FBJ. Lasers Principles and Applications. United Kingdom: Prentice Hall International Ltd.; 1987.
[67] Sanchez-Rubio Antonio. Wvelength Beam-Combined Laser Diode Arrays, Technical Notes, R\&D 100. Lincoln Laboratory, Massachusetts Institute of Technology; 2012 (www.II.mit.edu).

[68] Fan TY. Laser beam combining for high-power high-radiance source. IEEE J. Sel. Top. Quantum Electron. 2005;11:567-77.

[69] R.J. Lang, Advanced high brightness lasers, laser and electro-optics society annual meetings, in: Proceedings of the 10th IEEE Annual Meeting Conference 1997, LEOS'97, vol. 12, San Francisco, CA, USA, 1997. http://dx.doi.org 10.1109/LEOS.1997.645526.

[70] Y. Hirano, Diode-Pumped High Power ( > 100 W), High-Brightness Solid-state Lasers, IEEE, 1999, pp. 908-909.

[71] Abdolvand A, Wilcox KG, Kalkandjiev TK, Rafailov EU. Conical refraction Nd: KGd $\left(\mathrm{WO}_{4}\right)_{2}$ laser. Opt. Express 2010;18(3):2753-9.

[72] A.D. Yanson, B. Qin, O.P. Kowalski, S.D. McDougall, J.H. Marsh, Novel HighBrightness Laser Diodes at $830 \mathrm{~nm}$, IEEE, pp. 585-586, 0-7803-9556-5/06/ $\$ 20.00$.

[73] G. Machavariani, Y. Lumer, I. Moshe, I. Meir, S. Jackel, N. Davidson, MachZehnder-Like Interferometer in a High-Power Laser.

[74] Hanxuan Li, Truchan T, Brown D, Pryor R, Pandey R, Reinhardt F, Mott J, Treusch G, Macomber S. Reliable high-efficiency high-brightness laser diode bars at $940 \mathrm{~nm}$. Opt. Laser Technol. 2004;36:327-9.

[75] M. Lei, Efficient Intracavity Beam, in: Mikhail Grishin (Ed.) Advances in SolidState Lasers, Development and Application, Croatia, 2010, Available at 〈www. intechopen.com>.

[76] Wang PY, Gheen A, Wang Z. Beam shaping technology for laser diode arrays, laser beam III. Proc. SPIE 2002;4770 0277-786x/02/\$15.00.

[77] Bergmann JP, Patschger A, Bastick A. Enhancing process efficiency due to high focusing with high brightness laser - applicability and constrains. Phys. Procedia 2011;12:66-74.

[78] P. Sprangle, A. Ting, J. Peñano, R. Fischer, B. Hafizi, High-Power Fiber Laser for Directed-Energy Applications, NRL Review, Featured Research, 2008, pp. 8999.

[79] H. Ishizuka, Y. Nakahara, S. Kawasaki, K. Sakamoto, A. Watanabe, N. Ogiwara, M. Shiho, Ultra-Brightness Microbeams: Consideration for Their Generation and Relevance to FEL, IEEE, 1993, pp. 1566-1568.

[80] Ronen E, Friesem AA, Davidson N. Passive Beam combining in compact slab lasers. IEEE J. Quantum Electron. 2010;46(1):76.

[81] Bluem H, Todd AMM, Cole MD, Ratheke J, Schultheiss T. Recent advances in high-brightness electron gun at AES. Nucl. Instrum. Methods Phys. Res. - Sect. A 2003;507:215-9.

[82] M. Zolotorev, E.D. Commins, S. Heifets, F. Sannibale, Proposal for a highbrightness pulsed electron source, in: Contribution to Workshop on the Physics and Applications of High Brightness Electron Beams (PAHBEB 2005), 9-15 October 2005, Erice, Sicily, Italy, SLAC-PUB-12160, 2005, pp. $1-7$.

[83] Shaw MJ, Ross IN, Hooker CJ, Dodson JM, Hirst GJ, Lister JMD, Divall EJ, Kidd AK, Hancocks S, damerell AR, Wyborn BE. Ultrahigh-brightness KrF laser system for fast ignition studies. Fusion Eng. Des. 1999;44:209-14.

[84] Siew Ronian. Corrections to classical radiometry and the brightness of stars. Eur. J. Phys. 2008;29:1105.

[85] Bastien Fabienne A, Stassun Keivan G, Basri Gibor, Pepper Joshua. An observational correlation between stellar brightness variations and surface gravity. Nature 2013;500:427-30.

[86] Bastien Fabienne A, Stassun Keivan G, Basri Gibor, Pepper Joshua. A brighter method for measuring the surface gravity of distant stars. Nat. Lett. 2013;500:427-30. 